\title{
Study of complications following various thyroidectomy procedures: a tertiary care centre experience in South India
}

\author{
Madhu C.P. ${ }^{1}$, Ankit Raj ${ }^{2}$ \\ ${ }^{1}$ Dr. Madhu C.P., Professor and HOD, ${ }^{2}$ Dr Ankit Raj; Resident, Department of General Surgery, JSS Medical College, \\ Mysuru, Karnataka, India
}

Corresponding Author: Dr. Ankit Raj, Resident, Department of General Surgery, JSS Medical College, Mysuru, Karnataka, India. E-mail: ankit.kmc89@gmail.com

\begin{abstract}
Introduction: Thyroid surgeries comprise one of the more commonly performed procedures by a general surgeon. Today most of the complications of thyroid surgery are related to either metabolic derangements or injury to the recurrent laryngeal nerve injury. Other complications include superior laryngeal nerve injury, infection, airway compromise, and bleeding. At present, mortality for this procedure approaches $0 \%$ and overall complication rate is less than $3 \%$. Nonetheless, major complications of thyroidectomy (i.e. compressive hematoma, recurrent laryngeal nerve palsy and hypoparathyroidism) are still fearful complications and account for a significant percentage of medico-legal claims. Patients volume and surgical skill play an important role in reducing the risk of complications. Knowledge about the clinical profiles of thryoidectomy cases and understanding the post thyroidectomy complications are an important milestone in public health. Aims \& Objectives: This study intends to identify the incidence of the complications following various thyroidectomy surgeries performed at JSS Hospital, Mysuru and the effect of timely and prompt recognition and intervention in minimizing morbidity. Methodology: This clinical study was conducted between October 2017 and September 2019 and included 70 consecutive patients undergoing various thyroidectomy procedures, at JSS Hospital, Mysuru. All cases were followed up for a period of 6 months. Results: In our prospective observational study 70 cases of patients with thyroid swellings underwent various thyroidectomy procedures. The incidence of thyroid diseases is more common in females than in males. The peak age group in which patients presented to the hospital was in the $4^{\text {th }}$ decade . The most common histopathological diagnosis was Nodular Colloid Goitre. The most common surgery performed in was Total thyroidectomy in 45 cases out of 70 . Transient hypocalcemia was the most common post- operative complication occurring in about 13 out of 70 cases.
\end{abstract}

Keywords: Complications; Hypocalcaemia; Laryngeal nerve; Thyroid

\section{Introduction}

Thyroid disorders form one of the commonest causes in patients presenting with metabolic disturbances. Surgery forms a main part of the treatment modality offered to these patients. The first three successful thyroidectomies reported by William Warren Green in 1871 depicted that the procedure is never to be done to for the relief of deformity but only to save the life. Due to poor results and very high morbidity thyroidectomy procedures were banned by the French academy of Medicine in 1850 .

Due to improvement in the knowledge, experience and application of correct technique by 1909 when Theodore Kocher was given Nobel Prize, the mortality for thyroidectomy procedures for conditions like goiter reduced to less than $1 \%$. The current procedure of

Manuscript Received: $18^{\text {th }}$ October 2019

Reviewed: $28^{\text {th }}$ October 2019

Author Corrected: $5^{\text {th }}$ November 2019

Accepted for Publication: $8^{\text {th }}$ November 2019

Surgical Update: International Journal of Surgery \& Orthopedics thyroidectomy is mainly based on the principle explained by Thompson etal in 1973 who is regarded as a pioneer in the field of thyroid surgeries in Australia. Even though the post thyroidectomy complications have become rare these days, when occurring they can be quite devastating and sometimes may result in danger to life. Some of the major complications which may occur post procedure are postoperative bleeding, hematoma formation resulting in airway obstruction, thyroid storm, hypocalcemia and injuries to the laryngeal nerves.

Thyroidectomy procedures are the third most common cause of RLN injuries resulting in bilateral vocal fold immobility. Complications like recurrent laryngeal nerve injuries and permanent hypocalcaemia cause patients having a higher morbidity and may require lifelong treatment, mutltiple surgical procedures and rehabilitation resulting in poor quality of life and increased medical

Available online at: www.medresearch.in 322 | P a g e 
expenses. In modern era the main focus with respect to thyroidectomy procedures is on the postoperative morbidity with specific emphasis on preventing injuries to vital structures like RLN and parathyroid glands.

Total thyroidectomy can result in injury to all four parathyroid glands causing hypocalcemia or bilateral RLN injury when compared to unilateral lobectomy or hemithyroidectomy.

Henceforth it is important to assess the incidences of postoperative complications and to identify potential factors for these morbidities.

This information is helpful in preoperative counseling of the patients.

This study aims to identify the complications occurring post various thyroidectomy procedures and the importance of preoperative preparation of the patient, correct surgical technique and early identification of the postoperative complications and their treatment to reduce the morbidity and improve quality of life in such patients.

\section{Materials and Methods}

Study Design: Prospective Observational study

Study place: JSS Hospital, Mysuru, Department of General Surgery

\section{Study Duration: October 2017 To APRIL 2019}

\section{Sampling technique and study population:}

Convenient Sampling: All patients undergoing total thyroidectomy in JSS Hospital during the study period will be included in the study subject to their consent and exclusion criteria.

\section{Inclusion criteria}

1) Patients consenting to participate in the study

2) All the patients undergoing various thyroidectomy procedures performed at JSS Hospital during the study period

\section{Original Research Article}

\section{Exclusion criteria}

1) Patient with history of previous thyroid surgery

2) Patient who has adverse factors affecting wound healing like severe malnutrition, immuno suppressed states, post radiation, long term steroid therapy etc.

3) Patient who were lost to follow up

\section{Study setting and Method of collection of data}

- Name, age, sex, IP number of all patients included in study was documented

- A detailed history was taken and a physical examination done for all the patients

- Routine investigations like thyroid function tests, indirect laryngoscopy (IL), serum calcium alongwith ultrasonography of neck and FNAC were done.

- Intra operative documentation of duration of surgery, bleeding was done.

- Post operatively the patients were monitored for any complications like oozing, neck swelling, stridor, hoarseness of voice, features of hypoparathyroidism and wound infection.

- Apart from routine post operative investigations, serum calcium was checked routinely 24 hour post surgery and corrected for serum albumin levels. A fall in corrected serum calcium concentration below $8 \mathrm{mg} / \mathrm{dL}$, and /or the need for calcium supplementation was defined as temporary hypoparathyroidism. The need for oral vitamin D and / or calcium supplements six months following surgery to maintain a normal serum calcium concentration was taken as permanent hypoparathyroidism.

- Post-operative Indirect Laryngyoscopy was done for patients with hoarseness of voice on the 5th postoperative day. A temporary palsy is one which recovers within six months. Permanent recurrent laryngeal nerve injury is defined as vocal cord palsy, diagnosed by indirect laryngoscopy, which lasts for more than six months post operatively. Follow up serum thyroid stimulating hormone were monitored in second post op week, and then at six months.

- The data collected was analyzed using descriptive statistical principles

\section{Results}

In the current study an in depth analysis of the patients who underwent various thyroidectomy procedures JSS Hospital, Mysuru between October 2017 and April 2019 was done. In this study, the peak age group of the patients belonged to the fourth decade of life i.e., 31-40 years age group.

Second most common age group was 41-50 years comprising 17 patients. (24.2\%). Lesser number of cases were seen in extremes of age. The youngest patient was 19 years old and the oldest patient was 86 years old (Table 1). 
Original Research Article

Table-1: Age wise distribution.

\begin{tabular}{|c|c|c|}
\hline Age group (years) & No. of cases & Percentage \\
\hline $11-20$ & 01 & 1.4 \\
\hline $21-30$ & 14 & 20.5 \\
\hline $31-40$ & 20 & 24.2 \\
\hline $41-50$ & 17 & 17.1 \\
\hline $51-60$ & 12 & 2.8 \\
\hline $61-70$ & 02 & 4.2 \\
\hline $71-80$ & 03 & 1.4 \\
\hline $81-90$ & 01 & $\mathbf{1 0 0}$ \\
\hline Total & $\mathbf{7 0}$ & \\
\hline
\end{tabular}

The female to male ratio was found to be 9.43: 5.7 .

The mode of presentation and swelling was the common symptom observed in 48 cases, whereas the occurrence of swelling along with insomnia was the least observed symptom (Table 2)

Table-2: Mode of presentation.

\begin{tabular}{|c|c|c|}
\hline Symptoms & No. of cases & Percentage \\
\hline Swelling & 48 & 68.6 \\
\hline Swelling + pain & 07 & 7.1 \\
\hline Swelling + voice change & 05 & 7.1 \\
\hline Swelling + weight loss & 05 & 2.9 \\
\hline Swelling + dyspnoea & 02 & 2.9 \\
\hline Swelling + weight gain & 02 & 1.4 \\
\hline Swelling + insomnia & 01 & 7.9 \\
\hline
\end{tabular}

Clinical diagnosis was performed in the study population, and multinodular goiter was the most common diagnosis found in 42 cases (Table 3 ).

Table-3: Clinical diagnosis.

\begin{tabular}{|c|c|c|}
\hline Diagnosis & No. of cases & Percentage \\
\hline Multinodular goiter & 42 & 60 \\
\hline Toxic Nodular Goitre & 02 & 2.85 \\
\hline Graves Disease & 02 & 2.85 \\
\hline Left Solitary Nodule & 10 & 14.3 \\
\hline Right Solitary Nodule & 14 & 20 \\
\hline
\end{tabular}

Table-4: Histopathological diagnosis.

\begin{tabular}{|c|c|c|}
\hline Diagnosis & No. of cases & Percentage \\
\hline Multinodular goiter & 14 & 20 \\
\hline Diffuse colloid goiter & 06 & 22.9 \\
\hline Nodular colloid Goitre & 16 & 4.3 \\
\hline Adenomatoid Goitre & 03 & 1.4 \\
\hline Graves Disease & 01 & 17.1 \\
\hline Hashimoto's thyroiditis & 12 & 4.3 \\
\hline Papillary carcinoma & 3 & 4.3 \\
\hline Follicular carcinoma & 3 & 15.7 \\
\hline Follicular adenoma & 11 & 1.4 \\
\hline Hurthle Cell Adenoma & 01 & \\
\hline
\end{tabular}


Original Research Article

In the present study Nodular colloid goiter was the most common diagnosis found on histopathological examination which accounted for 16 out of the 70 cases (22.9\%). Multinodular goiter was found in 14 patients (20\%), followed by Hashimoto's thyroiditis (17.1\%) and Follicular adenoma (15.7\%). Malignancy (papillary and follicular carcinoma, 4.3\% each) was identified in 6 cases and Follicular adenoma was confirmed in 11 cases (15.7\%) (Table 4).

Table-5: Operative procedure performed.

\begin{tabular}{|c|c|c|}
\hline Procedure & No. of cases & Percentage \\
\hline Total thyroidectomy & 44 & 62.8 \\
\hline Subtotal thyroidectomy & 02 & 2.9 \\
\hline Right Hemithyroidectomy & 14 & 14.3 \\
\hline Left Hemithyroidectomy & 10 & 20 \\
\hline
\end{tabular}

The commonest procedure performed during the present study was total thyroidectomy which was done in 44 cases (62.8\%). Subtotal thyroidectomy was the least commonly performed procedure, done in 2 of these patients. Hemithyroidectomy was the second most commonly performed procedure done in a total of 24 cases $(34.3 \%)$ (Table 5).

Table-6: Incidence of post-operative complications.

\begin{tabular}{|c|c|c|}
\hline Post-operative complication & No. of cases & Incidence in percentage \\
\hline Bleeding & 2 & 2.86 \\
\hline Haematoma & 1 & 1.43 \\
\hline Transient Hypocalcemia & 9 & 2.85 \\
\hline Permanent Hypocalcemia & 2 & 1.43 \\
\hline RLN paralysis & 1 & 0 \\
\hline SLN paralysis & 0 & 0 \\
\hline Thyrotoxic storm & 0 & 0 \\
\hline Airway obstruction & 0 & 0 \\
\hline Seroma & 0 & 1.43 \\
\hline Wound infection & 1 & 0 \\
\hline Hypothyroidism & 0 & 0 \\
\hline Recurrent hyperthyroidism & 0 & 0 \\
\hline Hypertrophic scar/keloid & 0 & 0 \\
\hline
\end{tabular}

Hypocalcemia was the most common complication observed in the present study. Transient hypocalcemia was seen in 9 cases $(12.9 \%)$ and Permanent hypocalcemia was seen in 2 cases $(2.85 \%)$. Excessive intraoperative bleeding was noted in two cases $(2.86 \%)$ of which one patient was given postoperative blood transfusion in view of anaemia. One patient had post-operative hematoma formation for which wound re exploration was done and bleeder was identified and ligated. Wound infection was documented in one case on $4^{\text {th }}$ post-operative day. Right RLN paralysis due to iatrogenic injury was seen in one patient. Diagnosis was confirmed by post operative IDL on $5^{\text {th }}$ POD. Other complications such as thyroid storm, seroma formation, hypertrophic scar/keloid and recurrent hyperthyroidism were not observed during the present study (Table 6).

Table-7: Interventional procedures performed.

\begin{tabular}{|c|c|c|}
\hline Procedure & No. of cases & Percentage \\
\hline Oral Calcium+ Vitamin D+ Calcium gluconate i.v & 6 & 8.57 \\
\hline Oral calcium Supplements & 5 & 7.14 \\
\hline Antibiotic therapy & 1 & 1.43 \\
\hline Blood Transfusion & 1 & 1.43 \\
\hline Steroids + Speech Therapy & 1 & 80 \\
\hline No intervention required & 56 & 1.43 \\
\hline
\end{tabular}




\section{Original Research Article}

Intravenous Calcium gluconate along with Oral Calcium and Vitamin D therapy were administered for 6 cases in this study for patients showing features suggestive of clinical and biochemical hypocalcemia. These patients were advised to continue oral Calcium supplements at discharge and were followed up with serum calcium levels at 6 months. Oral calcium supplements were prescribed for 5 of our patients who were hypocalcemic but did not show any clinical signs of hypocalcemia. These patients were given supplements for 15 days at time of discharge and were reviewed in OPD. One patient was given 1 unit of postoperative blood transfusion in view of excessive bleeding intraoperatively. Wound site infection was observed in 1case and was successfully managed with local wound dressing and oral antibiotics for 1 week. One patient developed RLN paralysis post operatively and diagnosis was confirmed via Indirect larngyoscopy on $5^{\text {th }}$ POD. Patient was managed conservatively with steroids and speech therapy. After 6 months of follow up patient has recovered completely. One patient who developed hematoma post operatively with tense wound site and dry drain was taken for immediate re exploration of the wound and bleeding vessel was identified and ligated. The number of patients recovered without any complications were 56 out of $70(80 \%)$ and no intervention was required for these cases (Table 7).

In cases of total thyroidectomy, the complication of permanent hypocalcemia was the most common (Table 8).

Table-8: Different Operative Procedures and associated complications.

\begin{tabular}{|c|c|c|c|c|c|c|c|c|c|c|c|c|}
\hline \multirow[b]{2}{*}{ Type of surgery } & \multicolumn{12}{|c|}{ Type of complication } \\
\hline & 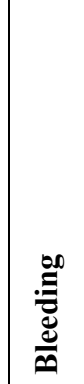 & 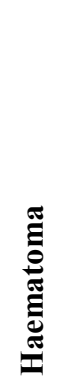 & 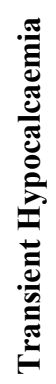 & 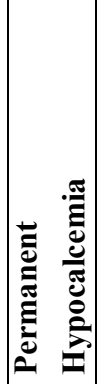 & 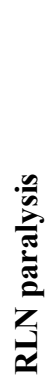 & 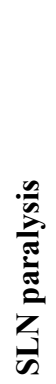 & 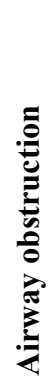 & 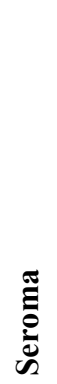 & 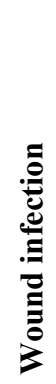 & 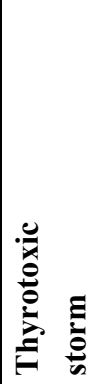 & 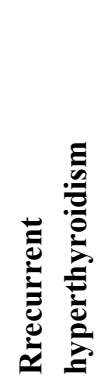 & 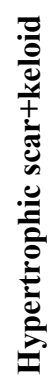 \\
\hline Total thyroidectomy & 0 & 0 & 9 & 2 & 1 & 0 & 0 & 0 & 0 & 0 & 0 & 0 \\
\hline Subtotal Thyroidectomy & 1 & 1 & 0 & 0 & 0 & 0 & 0 & 0 & 0 & 0 & 0 & 0 \\
\hline Right Hemithyroidectomy & 0 & 0 & 0 & 0 & 0 & 0 & 0 & 0 & 0 & 0 & 0 & 0 \\
\hline Left Hemithyroidectomy & 0 & 0 & 0 & 0 & 0 & 0 & 0 & 0 & 1 & 0 & 0 & 0 \\
\hline
\end{tabular}

Table-9: Duration of hospital stay post-surgery.

\begin{tabular}{|c|c|c|}
\hline Postoperative hospital stays (days) & No. of cases & Percentage \\
\hline $1-4$ & 30 & 42.9 \\
\hline $5-7$ & 34 & 48.6 \\
\hline $8-10$ & 01 & 1.43 \\
\hline$>10$ & 03 & 4.3 \\
\hline
\end{tabular}

34 patients $(48.6 \%)$ had a post operative hospital stay between 5 to 7 days. 30 patients had a hospital stay less than 4 days. In the present study the mean post-operative stay for the patients following surgery was about 5.3 days. One patient had a hospital stay of 8-10 days and 3 patients stayed in hospital more than 10 days with maximum being for a period of 21 days as patient had severe hypocalcemia post operatively (Table 9). Various complications such as bleeding, hematoma formation, Hypocalcaemia, RLN and SLN injuries, wound infection etc. were the attributable cause for this increased duration of stay post operatively.

\section{Discussion}

Thyroid gland is one of the most important structures in the body which is present in the region of neck. The neck region is traversed by various vital structures such as vessels and nerves which are closely related to the gland. Any injury occurring to these structures may result in increased morbidity or mortality to the patient. Hence it is imperative that extreme precaution should be taken intraoperatively during the dissection of the gland. In the current study Data collected from the 70 patients was 


\section{Original Research Article}

analysed with respect to several parameters and conclusions drawn. A comparative analysis with other studies was also made. In the present study, the youngest patient was 19 years old whereas the oldest patient was 86 years old. The average age for the subjects participating in the study was.

\section{Taking gender prevalence, the female to male ratio was 9.43:5.7}

In the current study, 48 out of 70 patients presented with a single complaint of swelling in the neck. 7 patients presented with pain and swelling, 5 patients had associated voice changes and only 1 patient presented with insomnia. Multinodular goiter the most common clinical diagnosis in this study and was seen in 42 patients $(60 \%)$. Second most common diagnosis was solitary nodule of thyroid seen in 24 patients (34.3\%). In the present study, 6 cases were found to be malignant on histopathological examination. A comparative analysis of the collected data was also done with other studies (Table 10).

Table-10: Comparative analysis of histopathological diagnosis with other studies.

\begin{tabular}{|c|c|c|}
\hline \multirow{2}{*}{ Studies } & \multicolumn{2}{|c|}{ Histopathological diagnosis } \\
\cline { 2 - 3 } & Malignant & Benign \\
\hline Sasson [1] & $52 \%$ & $48 \%$ \\
\hline Rix [2] & $10 \%$ & $90 \%$ \\
\hline Sakorafas [3] & $27 \%$ & $83 \%$ \\
\hline Present study & $8.57 \%$ & $91.43 \%$ \\
\hline
\end{tabular}

Table-11: Wound infection: comparative analysis.

\begin{tabular}{|c|c|c|c|}
\hline Clinical Studies & Year & Number of cases & Percentage \\
\hline Bergenfelz et al [4] & 2008 & 3660 & 1.6 \\
\hline Bhattacharya [5] & 2002 & 517 & 0.2 \\
\hline Rosato et al [6] & 2004 & 14,934 & 0.3 \\
\hline Current Study & 2019 & 70 & 1.43 \\
\hline
\end{tabular}

In the current study one patient who underwent left hemithyoidectomy had surgical site infection which was identified on POD $4^{\text {th }}$. Pus was drained and regular antiseptic dressings for healing by secondary intention and oral antibiotics were given following which patient recovered successfully. The complication rates are comparable with Bergenfelz [4] series (Table 11).

Table 12: Hypocalcemia: comparative analysis.

\begin{tabular}{|c|c|c|c|}
\hline Studies & Year & Number of cases & Percentage \\
\hline Rosato et al [6] & 2004 & 14,934 & 8.3 \\
\hline Bhattacharya [5] & 2002 & 517 & 6.2 \\
\hline Erbil.Y et.al [7] & 2007 & 3250 & 6.6 \\
\hline Current study & 2019 & 70 & 12.9 \\
\hline
\end{tabular}

In our current study transient hypocalcemia was the most common complication amounting to around 12.9 percent $(9$ out of 70 cases) of the patients and 2 patients developed permanent hypocalcemia (Table 12). Among these 11 patients 2 patients developed carpo pedal spasm and other features. Out of these 11 cases 4 were administered IV Calcium Gluconate along with oral supplements. Rest 4 patients were managed with oral supplements. According to the study done by Richmond [8] postoperative hypocalcemia rate was found to be $14 \%$.

Table-13: Hemorrhage: comparative analysis.

\begin{tabular}{|c|c|c|c|}
\hline Studies & Year & Number of cases & Percentage \\
\hline Rosato et al.[6] & 2004 & 14,934 & 1.2 \\
\hline Bhattacharya[5] & 2002 & 517 & 1.1 \\
\hline Bergenfelz et al[4] & 2008 & 3660 & 2.1 \\
\hline Current Study & 2019 & 70 & 2.85 \\
\hline
\end{tabular}

Surgical Update: International Journal of Surgery \& Orthopedics

Available online at: www.medresearch.in 327 | P a g e 
Original Research Article

In the current study out of 70 cases hemorrhage was noted in 2 patients (Table 13). In one patient subtotal thyroidectomy was done whereas left hemithyroidectomy was done for the other case. In one of the cases patient was taken up for wound reexploration in view of expanding hematoma. The bleeding vessels were identified and ligated. Wound was closed with drain insitu. Patient recovered well.

Table-14: Recurrent laryngeal nerve palsy: comparative analysis.

\begin{tabular}{|c|c|c|c|}
\hline Studies & Year & Number of cases & Percentage \\
\hline Rosato et al [6] & 2004 & 14,934 & 2 \\
\hline Bhattacharya [5] & 2002 & 517 & 0.7 \\
\hline Erbil.Y et. al [7] & 2007 & 3250 & 1.8 \\
\hline Current Study & 2019 & 70 & 1.43 \\
\hline
\end{tabular}

In our current study the incidence of RLN injury was $1.43 \%$ (Table 14). This value is comparable with the previously done studies and is within acceptable range. As per the study done by Steurer [9] the incidence of RLN palsy in benign thyroid nodule, thyroid malignancy and hyperparathyroidism groups are as $3.4 \%, 7.2 \%$, and $2.5 \%$ respectively. In the present the patient was a 67-year-old female on antipsychotic drugs. Intraoperatively thyroid was adherent to the trachea and longer duration of surgery coupled with extensive dissection resulted in injury to RLN. The diagnosis was confirmed by a postoperative indirect laryngyoscopy. Patient was treated conservatively with steroids and speech therapy. Patient recovered completely in 2 weeks. Other 56 cases recovered well without any complications. All the patients were followed up to a period of 6 months irrespective of status at the time of discharge.

\section{Limitations}

- Patients participating in the study were mostly from areas in and around Mysuru.

- The patients were followed up only upto 6 months post- operatively. Hence a further study with a larger sample size representing actual population of the region with longer post- operative follow up is required to accurately assess the incidence and reduce bias in the results

\section{Conclusion}

The key factors in reducing the complications post thyroid surgeries can be attributed to Careful dissection, ensuring hemostasis, and a clear knowledge of the regional anatomy. Parathyroids, RLN and EBSLN should be preserved in thyroidectomy procedures. In order to identify and preserve RLN palpatory method, inferior approach, superior and lateral approaches may be used. Inability to identify the nerve intraoperatively may result in high risk of damage causing increased morbidity.

Accidental removal of the parathyroid gland or injury to the vascular pedicle may result in transient or permanent hypoparathyroidism, The key step is to dissect closer to the capsule of thyroid gland and to ligate the capsular branches meticulously to avoid injury to main trunk of inferior thyroid artery. Incidence of other complications such as flap edema, surgical site infection, hypoparathyroidism and External branch of superior laryngeal nerve are also less.

\section{What this study adds to the existing knowledge?}

The study describes the similar incidence of complications after various thyroidectomy procedures and the key areas to be stressed upon to minimize the morbidity.

\section{Author's Contribution}

Dr. C.P. Madhu: Formulated the aims \& objectives with study design and supervised for study

Dr. Ankit Raj: Contributed to the preparation of the manuscript, collection of data and its analysis

Funding: No funding sources

Conflict of interest: None declared

Ethical Approval: This study was approved by the Institutional Ethics Committee

\section{References}

1. Sasson AR, Pingpank JF Jr, Wetherington RW, Hanlon AL, Ridge JA. Incidental parathyroidectomy during thyroid surgery does not cause transient symptomatic hypocalcemia. Arch Otolaryngol Head Neck Surg. 2001; 127 (3): 304-308. doi: 10.1001/archotol.127.3.304.

2. Rix TE, Sinha P. Inadvertent parathyroid excision during thyroid surgery. Surgeon. 2006;4(6):339-342. doi: 10.1016/s1479-666x(06)80108-3.

3. Sakorafas GH, Stafyla V, Bramis C, Kotsifopoulos N, Kolettis T, Kassaras G. Incidental parathyroidectomy during thyroid surgery: an underappreciated complication of thyroidectomy. World J Surg. 2005;29(12):1539-1543. doi: 10.1007/s00268-005-0032-y. 
4. Bergenfelz A, Jansson S, Kristoffersson A, Mårtensson H, Reihnér E, Wallin G, et al. Complications to thyroid surgery: results as reported in a database from a multicenter audit comprising 3,660 patients. Langenbecks Arch Surg. 2008;393(5):667-673. doi: 10.1007/s00423008- 0366-7. Epub 2008 Jul 17.

5. Bhattacharyya N, Fried MP. Assessment of the morbidity and complications of total thyroidectomy. Arch Otolaryngol Head Neck Surg. 2002;128(4):389-392. doi: 10.1001/archotol.128.4.389.

6. Rosato L, Carlevato MT, De Toma G, Avenia N. Recurrent laryngeal nerve damage and phonetic modifications after total thyroidectomy: surgical malpractice only or predictable sequence? World J Surg. 2005; 29(6):780-784. doi: 10.1007/s00268-005-7653-z.

\section{Original Research Article}

7. Erbil Y, Barbaros U, Işsever H, Borucu I, Salmaslioğlu A, Mete $\mathrm{O}$, et al. Predictive factors for recurrent laryngeal nerve palsy and hypoparathyroidism after thyroid surgery. Clin Otolaryngol. 2007; 32(1):32-37. doi: 10.1111/j.13652273. 2007.01383.x.

8. Steurer M, Passler C, Denk DM, Schneider B, Niederle $\mathrm{B}$, Bigenzahn W. et al. Advantages of recurrent laryngeal nerve identification in thyroidectomy and parathyroidectomy and the importance of preoperative and postoperative laryngoscopic examination in more than 1000 nerves at risk. Laryngoscope. 2002;112(1):124-133. doi: 10.1097/00005537-200201000-00022.

9. Richmond BK, Eads K, Flaherty S, Belcher M, Runyon D. Complications of thyroidectomy and parathyroidectomy in the rural community hospital setting. Am Surg. 2007;73(4):332-336.

\section{How to cite this article?}

Madhu C.P, Ankit Raj. Study of complications following various thyroidectomy procedures: a tertiary care centre experience in South India. Surgical Update: Int J surg Orthopedics.2019;5(5):322-329.doi:10.17511/ijoso.2019.105.03 